\title{
PPMXl Photometric Study of Four Open Cluster Candidates (IVANOV 2, IVANOV 7, IVANOV 9 AND HARVARD 9)
}

\author{
A. L. TADROSS ${ }^{1}$ AND R. BENDARY ${ }^{1}$ \\ ${ }^{1}$ National Research Institute of Astronomy and Geophysics, 11421 Helwan, Cairo, Egypt; altadross@yahoo.com
}

Received May 28, 2014; accepted July 19, 2014

\begin{abstract}
The astrophysical parameters of four unstudied open star cluster candidates; Ivanov 2, 7, 9, and Harvard 9; are estimated for the first time using the PPMXL database. The stellar density distributions and color-magnitude diagrams for each cluster are used to determine the geometrical structure (cluster center, limited radius, core and tidal radii, the distances from the Sun, from the Galactic center and from the Galactic plane). Also, the main photometric parameters (age, distance modulus, color excesses, membership, total mass, relaxation time, luminosity and mass functions) are estimated.
\end{abstract}

Key words: Galaxy: open clusters and associations - individual: Ivanov 2, Ivanov 7, Ivanov 9, Harvard 9 - astrometry - Stars: luminosity function - Mass function

\section{INTRODUCTION}

Open star clusters (OCs) are important celestial bodies in understanding star formation, initial mass function and stellar evolution theories. Gaburov and Gieles (2008) have compiled statistically significant samples of star clusters of known distance, age and metallicity. Color-magnitude Diagram (CMD) analysis through isochrones give us good estimates of the astrophysical parameters of the clusters, e.g., age, reddening and distance. In the last decades, many studies have been performed using different techniques; started from photographic photometry to the couple charge devices, CCDphotometry, and finally employing many isochrones models. The large amount of results, which produced in the literature are gathered in catalogs and databases, e.g., Webda and Dias. The present study depends mainly on the PPMXL database of Röser et al. (2010). The most important reason for using PPMXL database lies in the positions, proper motions of USNO-B1.02 and the Near Infrared (NIR) photometry of the Two Micron All Sky Survey (2MASS), which allowed us to search and study clusters within the disk of the Galaxy that is normally obscured by dust and gas clouds. Such databases help us to unravel star formation problems and answer questions about the spiral structure of the Milky Way Galaxy.

Our candidates are selected from among the unstudied OCs listed in Dias catalog. Figure 1 represents the clusters' images as taken from the LEDAS DSS image of Digitized Sky Surveys (http://www.ledas.ac.uk/). The only available information known about these clusters are the coordinates and the apparent diameters in arcmin. Equatorial and Galactic positions of our candidates with their apparent diameters are listed in Table 1. The quality of the downloaded data is taken into account and the estimated physical properties of each cluster are achieved by applying the same methodology.

Corresponding Author: A. L. Tadross
This paper is organized as follows. PPMXL data extraction is presented in Section 2, while the data analysis and parameter estimations are described in Sections 3. Finally, the conclusion of our study is in Section 4 .

\section{PPMXL DATa Extraction}

The main data used in this work has been extracted from the PPMXL catalog of Röser et al. (2010), which contains $\sim 910$ million objects, of which 410 million objects have photometric data from 2MASS catalog. PPMXL catalog combines the USNO-B1.0 database of Monet et al. (2003) and the 2MASS database of Skrutskie et al. (2006) yielding the largest collection of proper motions in the International Celestial Reference Frame (ICRF). USNO-B1.0 contains the positions of more than one billion objects taken photographically around 1960, which gives us an opportunity to distinguish between the members and background/field stars. While 2MASS survey conducted in the years 1997 to 2001 ; provides $\mathrm{J}(1.25 \mu \mathrm{m}), \mathrm{H}(1.65 \mu \mathrm{m})$, and $\mathrm{K}_{s}(2.17 \mu \mathrm{m})$ band photometry of millions of galaxies and nearly a half-billion stars (Carpenter 2001).

In PPMXL, we can find the mean positions and proper motions for all objects of brightest magnitudes down to $\mathrm{V} \sim 20 \mathrm{mag}$. Mean errors of the proper motions vary from $\sim 4$ mas/yr (milliseconds per year) for $\mathrm{J}<10 \mathrm{mag}$ to more than $\sim 10 \mathrm{mas} / \mathrm{yr}$ at $\mathrm{J}>16 \mathrm{mag}$ (Khalaj \& Baumgardt 2013). Before using the proper motion data in the PPMXL catalog, it is very important to understand the systematic and random errors of the proper motions in this catalog. Röser et al. (2010) have corrected the plate-dependent distortions of the proper motions during the construction procedure of the PPMXL catalog. They have pointed out that the magnitude and color dependent systematic errors in the PPMXL catalog are difficult to determine due to the absence of an independent reference on ICRS at fainter magnitude (Wu et al. 2011). 
Table 1

Equatorial, Galactic coordinates and the apparent diameters of the candidate clusters; obtained from DIAS catalog.

\begin{tabular}{|c|c|c|c|c|c|}
\hline Cluster & $\mathrm{Ra}^{h}{ }^{h} m s$ & Dec. $^{\circ} \quad \prime \prime \prime \prime$ & G. long. ${ }^{\circ}$ & G. lat. & $\mathrm{D}^{\prime}$ \\
\hline Ivanov 2 & 061553 & +141600 & 196.214 & -1.198 & 2.0 \\
\hline Ivanov 7 & 073040 & -151800 & 230.983 & 1.484 & 2.8 \\
\hline Ivanov 9 & 065943 & -040400 & 217.494 & -0.016 & 1.0 \\
\hline Harvard 9 & 153346 & -534000 & 325.616 & 1.899 & 6.0 \\
\hline
\end{tabular}

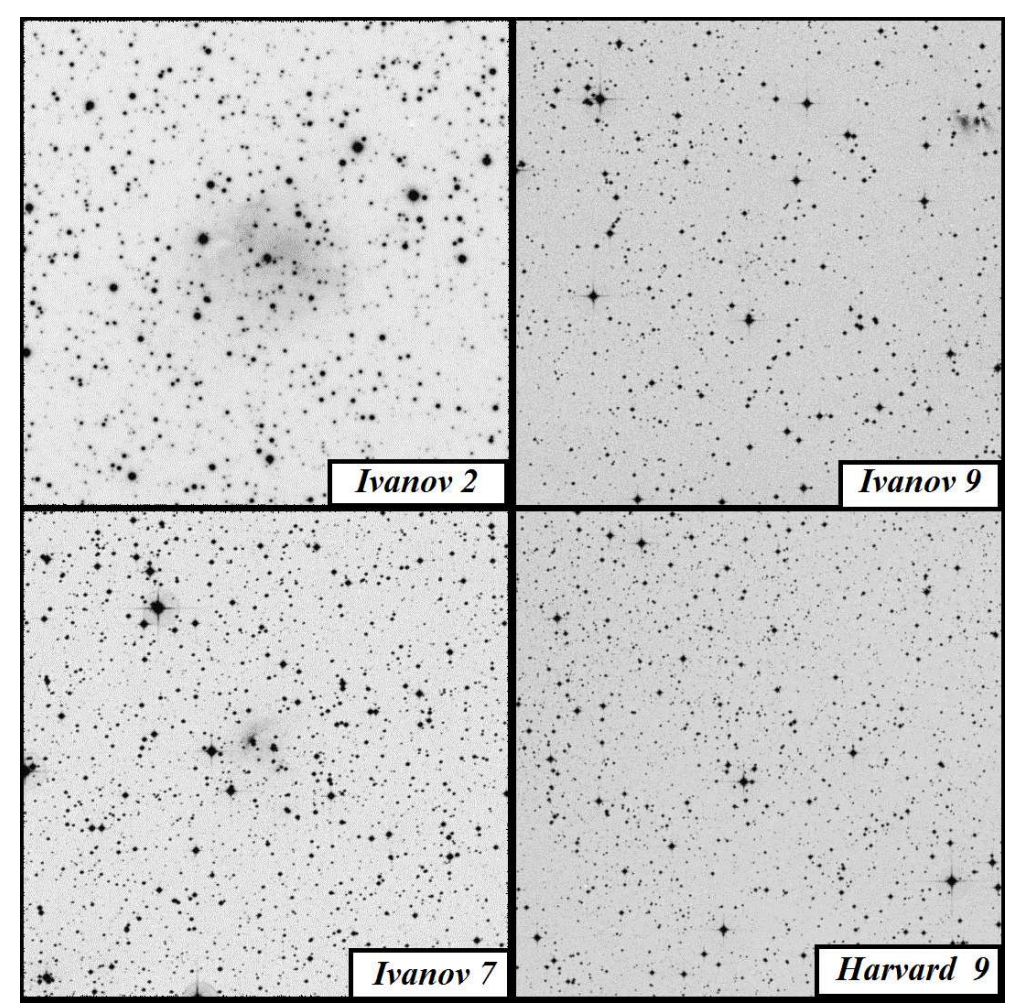

Figure 1. The images of candidate clusters, as taken from the LEDAS DSS Digitized Sky Surveys.

This survey has proven to be a powerful tool in the analysis of the structure and stellar content of open clusters (Bica et al. 2003; Bonatto \& Bica 2003). The photometric uncertainty of the 2MASS data is less than 0.155 at $\mathrm{K}_{s} \sim 16.5 \mathrm{mag}$ which is the photometric completeness for stars with $|b|>25^{\circ}$, Skrutskie et al. (2006).

It maybe noted that the candidate clusters are located near the Galactic plane $\left(|b|<2^{\circ}\right)$, therefore we expect significant foreground and background field star contamination. These clusters have medium central concentration, as seen from their images on the Digitized Sky Survey (DSS), see Figure 1. Their apparent diameters are less than 10 arcmin, and because it should reach the field background stars, hence the downloaded data are taken to be exceed the apparent diameters, i.e., about 10-15 arcmin.

In this context, to get net worksheet data for investigating clusters, the photometric completeness limit has been applied to the photometric pass-band 2MASS data to avoid over-sampling of the lower parts of the cluster's CMDs (cf., Bonatto et al. 2004). Stars with observational uncertainties $\geq 0.20$ mag have been removed. Pm vector point diagram (VPD) with distribution histogram of 2 mas/yr bins for ( $\mathrm{pm} \alpha \cos \delta)$ and $(\mathrm{pm} \delta)$ have been constructed as shown in Figure 2. The Gaussian function fit to the central bins provides the mean $\mathrm{pm}$ in both directions. All data that lie at mean $\pm 1 \sigma$ (where $\sigma$ is the standard deviation) can be considered as probable members. In addition, stellar photometric membership criteria are adopted based on the location of the stars within $\pm 0.1 \mathrm{mag}$ around the zero age main sequence (ZAMS) curves in the CMDs, (Clariá \& Lapasset 1986).

\section{Data Analysis}

\subsection{Cluster's Centers and Radial Density Profile}

The star-count has been applied to the candidate clusters' regions up to 10 arcmin of the adopted centers. Each area is divided into equal sized bins in right ascension $(\alpha)$ and declination $(\delta)$. The purpose of this counting process is to determine the maximum central density of the clusters. The clusters' centers are found 

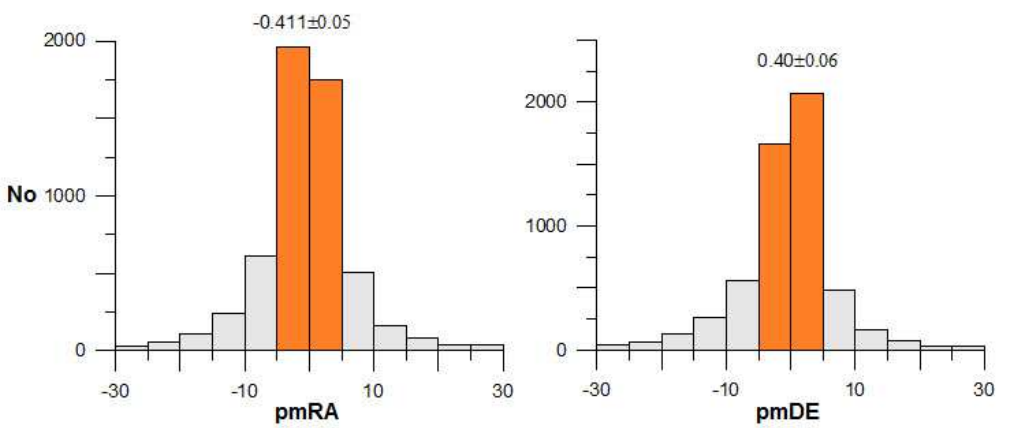

Ivanov 2
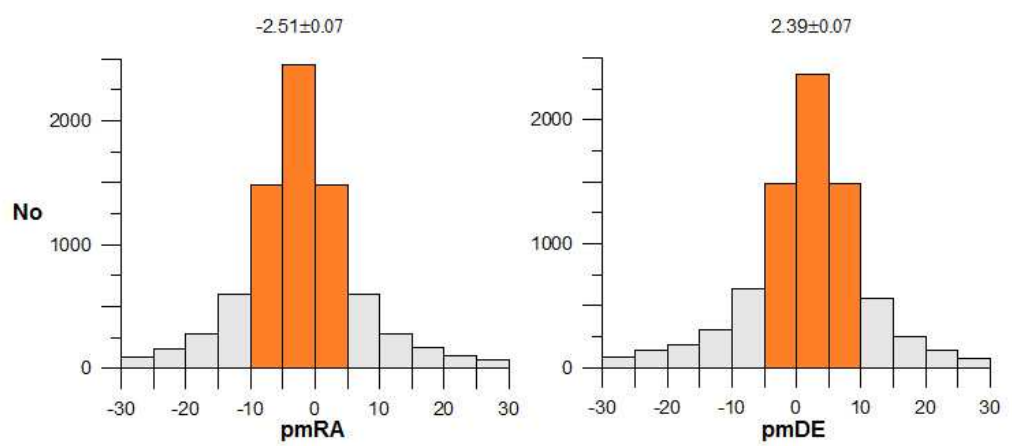

\section{Ivanov 7}
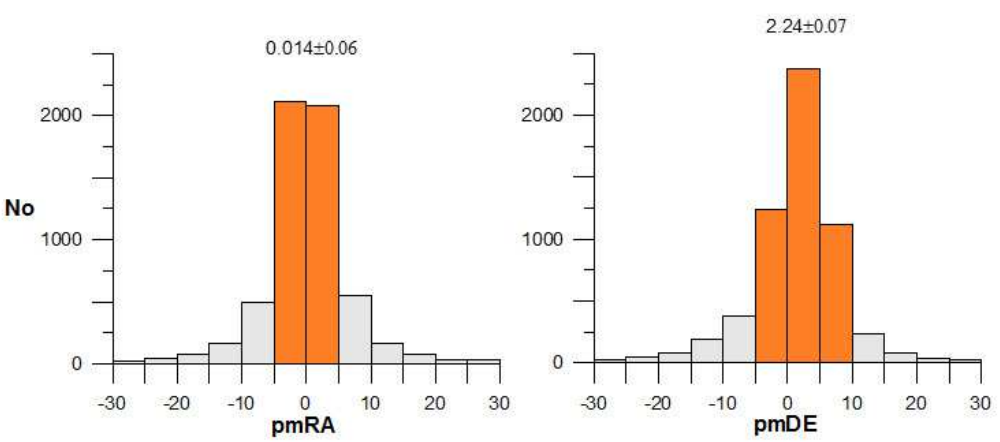

\section{Ivanov 9}
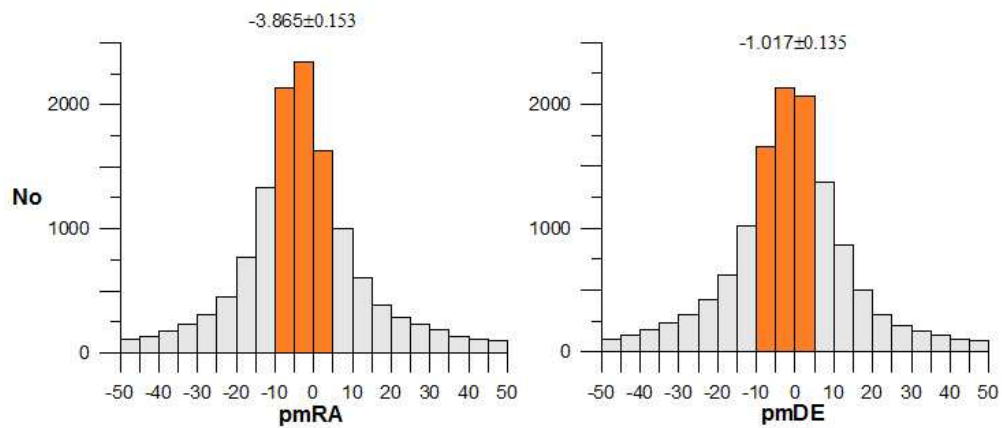

\section{Harvard 9}

Figure 2. Proper motion histograms of 5 mas/yr bins in right ascension and declination of the candidate clusters, the pm errors $\geq 4 \mathrm{mas} / \mathrm{yr}$ are excluded. The Gaussian function fit to the central bins provides the mean values in both directions as shown in each panel. The orange regions represent the limits taken of the membership. The standard error is taken to be $\pm 1 \sigma$ (the standard deviation of the Gaussian distribution).

by fitting Gaussian distribution function to the profiles of star counts in $\alpha$ and $\delta$ respectively; the standard errors are shown for each cluster as shown in Figure 3. The cluster center is defined as the location of the maximum stellar density of the cluster's area. The differ- ences between our estimated centers and those obtained by Webda are found to be very small, see Table 2 .

To establish the radial density profile (RDP) of the clusters under consideration, their areas are divided into central concentric circles with bin sizes $\mathrm{R}_{i} \leq 1$ ar- 
Table 2

The astrophysical parameters of the candidate clusters.

\begin{tabular}{|c|c|c|c|c|}
\hline Parameter & Ivanov 2 & Ivanov 7 & Ivanov 9 & Harvard 9 \\
\hline Center (Ra. ${ }^{h} \quad m{ }^{s}$ ) & 061548 & 073039 & 065947 & 153344 \\
\hline (Dec. $\left.{ }^{\circ}{ }^{\prime}{ }^{\prime \prime}\right)$ & +141551 & -151804 & -040400 & -533457 \\
\hline $\mathrm{R}_{\text {lim }}(\operatorname{arcmin})$. & 2.8 & 5.8 & 3.0 & 5.4 \\
\hline Diameter $(p c)$. & 2.36 & 6.96 & 2.18 & 4.55 \\
\hline Core radius (arcmin.) & 0.09 & 0.42 & 0.05 & 0.23 \\
\hline Tidal radius $(p c)$. & 12.20 & 11.40 & 12.60 & 16.50 \\
\hline Age $(M y r)$. & $(5 \sim 10)$ & $(400 \sim 800)$ & $(80 \sim 120)$ & $(200 \sim 400)$ \\
\hline $\mathrm{E}(\mathrm{J}-\mathrm{H})(\operatorname{mag})$. & 0.28 & 0.15 & 0.11 & 0.28 \\
\hline $\mathrm{E}(\mathrm{B}-\mathrm{V})($ mag. $)$ & 0.90 & 0.48 & 0.35 & 0.90 \\
\hline m-M (mag.) & 11.60 & 12.0 & 10.8 & 11.6 \\
\hline Distance (pc.) & $1445 \pm 65$ & $2065 \pm 95$ & $1250 \pm 60$ & $1445 \pm 65$ \\
\hline $\mathrm{R}_{g c}(k p c)$. & 8.60 & 8.65 & 8.23 & 6.10 \\
\hline $\mathrm{X}_{\odot}(p c)$. & 1390 & 1300 & 933 & -1195 \\
\hline $\mathrm{Y}_{\odot}(p c)$. & -405 & -1600 & -760 & -815 \\
\hline $\mathrm{Z}_{\odot}(p c)$. & -30 & 55 & -0.30 & 50 \\
\hline Membership (\#stars) & 500 & 590 & 665 & 1100 \\
\hline Total Lumin. of J-band (mag.) & -3.65 & -6.30 & -3.90 & -5.20 \\
\hline Total mass $\left(\mathcal{M}_{\odot}\right)$ & 580 & 470 & 645 & 1450 \\
\hline IMF slope & -2.46 & -2.73 & -3.0 & -2.31 \\
\hline Relax. time (Myr.) & 12.0 & 11.5 & 9.3 & 16.5 \\
\hline
\end{tabular}

cmin, from the cluster center. The number density, $\mathrm{R}_{i}$, in the $\mathrm{i}^{\text {th }}$ zone is calculated by using the formula of $\mathrm{R}_{i}$ $=\mathrm{N}_{i} / \mathrm{A}_{i}$ where $\mathrm{N}_{i}$ is the number of stars and $\mathrm{A}_{i}$ is the area of the $\mathrm{i}^{\text {th }}$ zone. The star counts of the next steps should be subtracted from the previous ones, so that we obtained only the amount of the stars within the relevant shell's area, not a cumulative count. The density uncertainties in each shell were calculated using Poisson noise statistics. Finally, we applied the empirical King model (1966), parameterizing the density function $\rho(r)$ as:

$$
\rho(r)=f_{b g}+\frac{f_{0}}{1+\left(r / r_{c}\right)^{2}}
$$

where $f_{b g}, f_{0}$ and $r_{c}$ are background, central star density and the core radius of the cluster respectively. The cluster's limiting radius can be defined at that radius which covers the entire cluster area and reaches enough stability with the background field density. It is noted that PPMXL catalog allows us to obtain reliable data on the projected distribution of stars for large extensions the clusters' halos. We can infer that open clusters appear to be somewhat larger in the nearinfrared than in the optical data, Sharma et al. (2006). Because of strong field stars contamination, it is not possible to completely separate all field stars from cluster members as shown for Ivanov 9 and Harvard 9. The limiting radius of a cluster can be described with an observational border, which depends on the spatial distribution of stars in the cluster and the density of the membership and the degree of field-star contamination. Figure 4 shows the RDP from the new centers of the clusters under consideration. The core radius and the background field density are estimated and shown for each cluster as well.

Finally, knowing the cluster's total mass (Section 3.4 ), the tidal radius can be calculated by applying the equation of Jeffries et al. (2001):

$$
R_{t}=1.46\left(M_{c}\right)^{1 / 3}
$$

where $R_{t}$ and $M_{c}$ are the tidal radius and total mass of the candidate clusters respectively. Nilakshi et al. (2002) noted that the halos' sizes are smaller for older systems.

\subsection{The PPMXL Photometry}

Using the PPMXL data, deep stellar analyses of the candidate clusters have been presented. The photometric data of PPMXL not only allow us to construct relatively well defined CM diagrams of the clusters, but also permit a more reliable determination of astrophysical parameters. In this paper, we used extraction areas having a radius of 10 arcmin, which are larger than the estimated limiting radius of the clusters. Because of the weak contrast between the cluster and the background field density, some inaccurate statistical results may be produced beyond the real limit of cluster borders (Tadross 2005).

The main astrophysical parameters of the clusters, e.g., age, reddening, distance modulus, can be determined by fitting the isochrones to the cluster CMDs. To do this, we applied several fittings on the CMDs of the clusters by using the stellar evolution models of Marigo et al. (2008) and Girardi et al. (2010) of Padova isochrones. It is worth mentioning that the assumptions of solar metallicity are quite adequate for young and intermediate age open clusters, which are close to the Galactic disk. So, Near-Infrared surveys are very useful for the investigation of such clusters. It is relatively less affected by high reddening from the Galactic plane. However, for a specific age isochrones, the fit should be obtained at the same distance modulus for both diagrams [J-(J-H) \& $\left.\mathrm{K}_{s^{-}}\left(\mathrm{J}-\mathrm{K}_{s}\right)\right]$, and the 

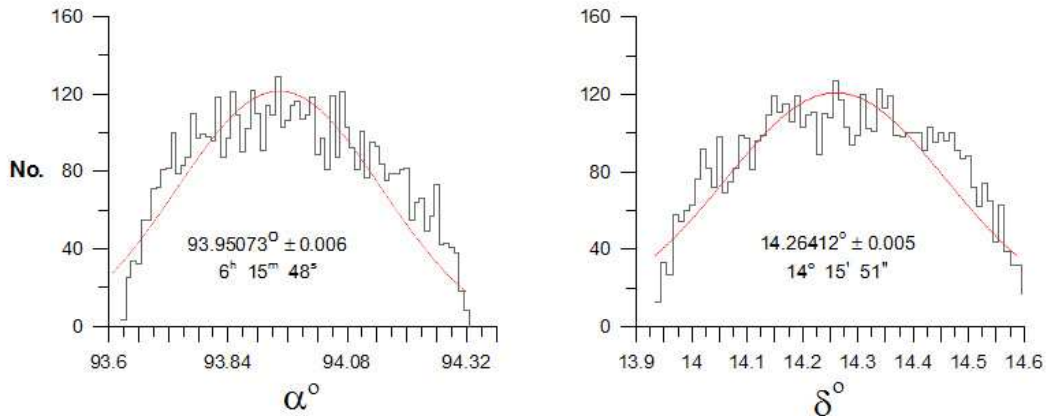

Ivanov 2
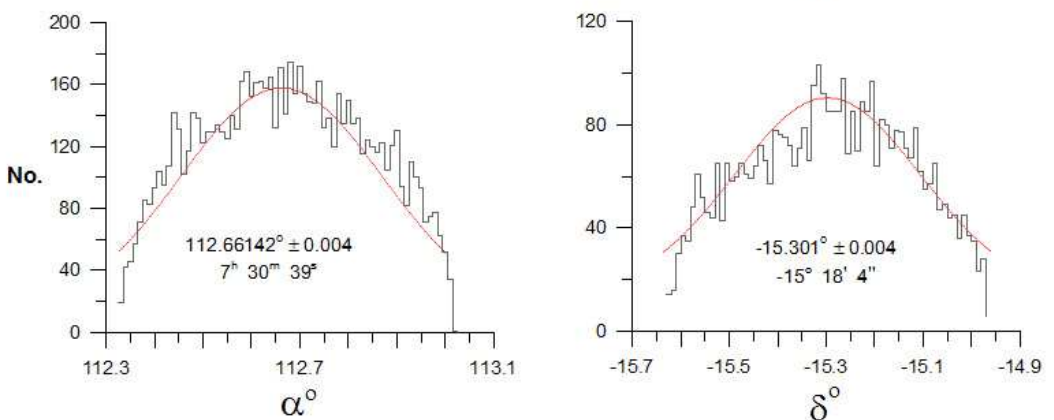

Ivanov 7
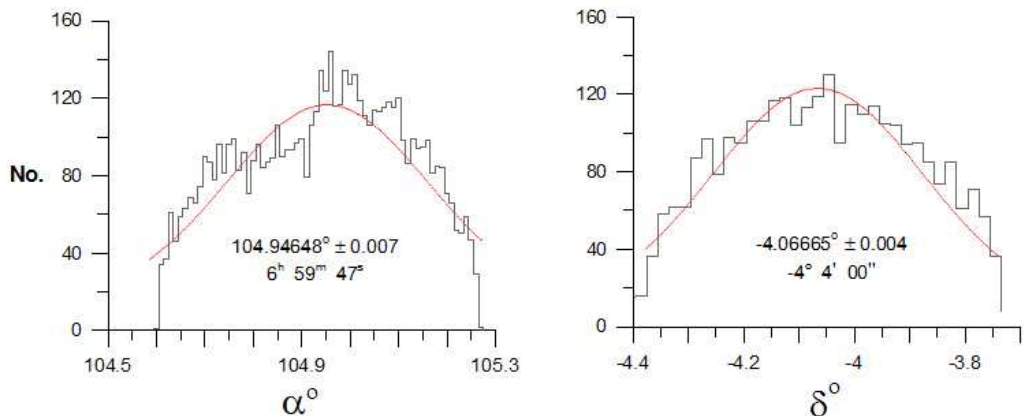

Ivanov 9
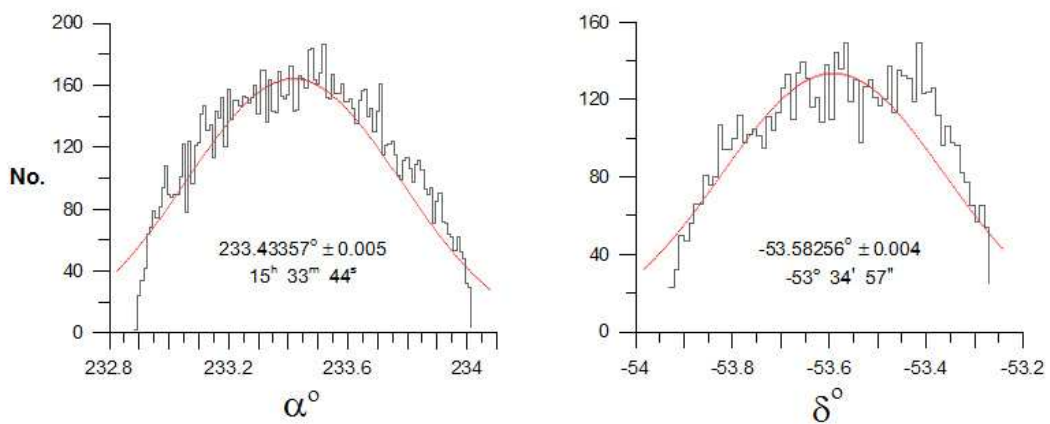

\section{Harvard 9}

Figure 3. The cluster center estimating of the candidate clusters. The Gaussian fit (red lines) provide the coordinates of highest density areas in $\alpha$ and $\delta$ for each cluster respectively. The center of symmetry about the peaks of $\alpha$ and $\delta$ is taken to be the position of the clusters' centers. The standard errors are shown for each cluster; it is taken to be $\pm 1 \sigma$ (the standard deviation of the Gaussian distribution).

color excesses should obey Fiorucci \& Munari (2003)'s relations for normal interstellar medium as shown in Figure 5. We note that, it is difficult to obtain accurate determinations of the astrophysical parameters due to the weak contrast between clusters and field stars. So, some ranges in age estimations are given. It might be mainly explained by the theoretical differences in adopting isochrones. Therefore, the clusters' ages are found to be (5-10); (400-800); (80-120); (200-400) Myr for Ivanov 2, 7, 9, and Harvard 9 respectively.

Reddening determination is one of the major steps in cluster compilation. Guiding by Schlegel et al. (1998) and Schlafly et al. (2011), the reddening of the clusters have been estimated. In this context, for color excess transformations, we used the coefficient ratios $\frac{A_{J}}{A_{V}}=0.276$ and $\frac{A_{H}}{A_{V}}=0.176$, which are derived 

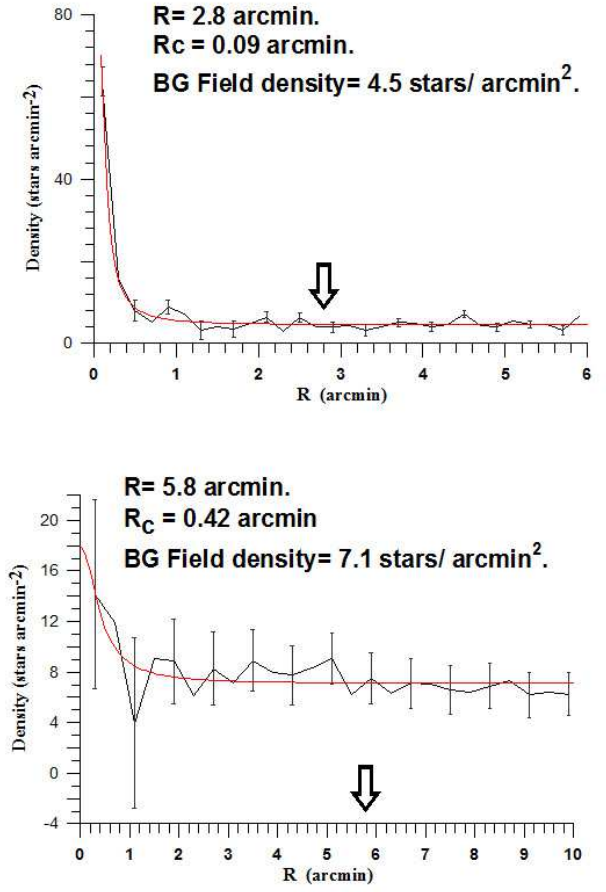

Ivanov 7
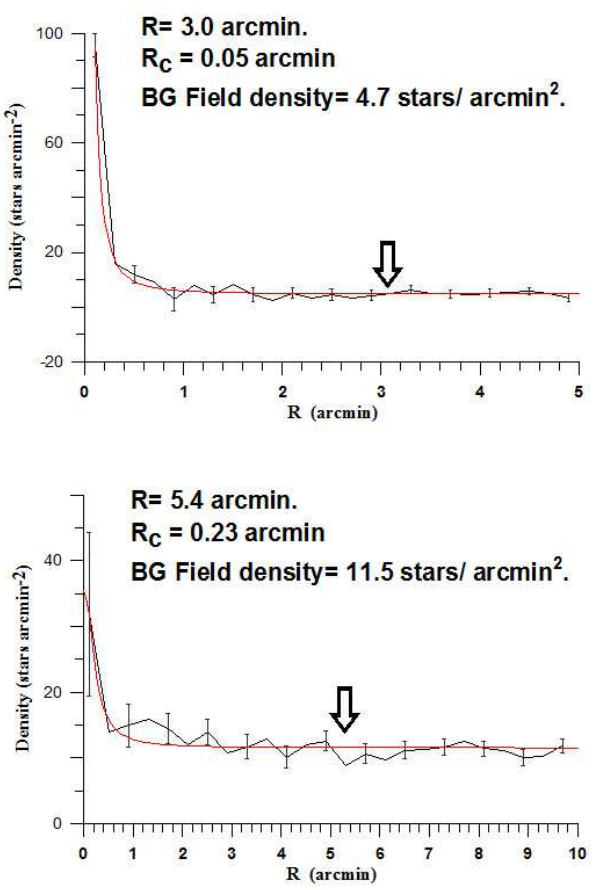

Figure 4. The radial density profile of the clusters under consideration. Clusters' limiting radius, core radius and the background field density are estimated and shown for each candidate clusters. The curved solid line represents the fitting of King model [12]. Error bars are determined from sampling statistics $\left[1 /(N)^{0.5}\right.$ where $\mathrm{N}$ is the number of stars used in the density estimation at that point].

from absorption ratios in Schlegel et al. (1998), while the ratio $\frac{A_{K_{s}}}{A_{V}}=0.118$ was derived from Dutra et al. (2002). Applying the calculations of Fiorucci \& Mu- nari (2003) for the color excess of 2MASS photometric system; we ended up with the following results: $\frac{E_{J-H}}{E_{B-V}}=0.309 \pm 0.130, \frac{E_{J-K_{s}}}{E_{B-V}}=0.485 \pm 0.150$, where $\mathrm{R}_{V}=\frac{A_{V}}{E_{B-V}}=3.1$. Also, we can de-redden the distance modulus using these formulae: $\frac{A_{J}}{E_{B}-V}=0.887, \frac{A_{K_{s}}}{E_{B}-V}=$ 0.322. then the distance of each cluster from the Sun $R_{\odot}$ can be calculated.

Under the assumption of $R_{g c \odot}=7.2 \pm 0.03 \mathrm{kpc}$ of Bica et al.(2006) which is based on updating the parameters of globular clusters, the estimated distances from the Galactic center $R_{g c}$ are estimated for each cluster. Also, the distance from the Galactic plane $\left(Z_{\odot}\right)$, and the projected distances in the Galactic plane from the Sun $\left(X_{\odot} \& Y_{\odot}\right)$ can be determined, see Table 2. For more details about the distance calculations, see Tadross (2011).

\subsection{Luminosity Functions}

It is difficult to determine the membership of a cluster using only the stellar RDP. It might be claimed that most of the stars in the inner concentric rings are quite likely members, whereas the external rings are more intensely contaminated by field stars. Therefore, the stars, which are closed to the cluster's center and near to the main-sequence (MS) in CMDs are taken to be the stellar membership of the clusters. These MS stars are very important in determining the luminosity, mass functions and the total mass of the investigated clusters. The measurements of the number of stars in a cluster with a given color and magnitude ranges are very important to understand the characteristic properties of the evolutionary stages of these objects.

For this purpose, we obtained the Luminosity Functions (LFs) of the four clusters by summing up the $\mathrm{J}$ band luminosities of all stars within the determined limiting radii for each cluster. Before building the LFs, we converted the apparent $\mathrm{J}$ band magnitudes of possible member stars into the absolute magnitude values using the distance moduli of the clusters. We constructed the histogram sizes of LFs to include a reasonable number of stars in each absolute $\mathrm{J}$ magnitude bins for the best counting statistics. The total LFs of the cluster are found to be $-3.65,-6.30,-3.90$ and $-5.20 \mathrm{mag}$ for Ivanov 2, 7, 9, and Harvard 9 respectively; see Figure 6.

\subsection{Mass Functions, Total Masses and Dynamical States}

The mass functions (MFs) of the four clusters are built using the theoretical evolutionary tracks and their isochrones with different ages. The masses of possible cluster members were derived from the polynomial expression developed by Marigo et al. (2008) and Girardi et al. (2010) with solar metallicity. The LF and MF are correlated to each other according the known Mass-luminosity relation. The accurate determination of both of them (LF \& MF) suffers from the field star contamination, membership uncertainty, and mass seg- 

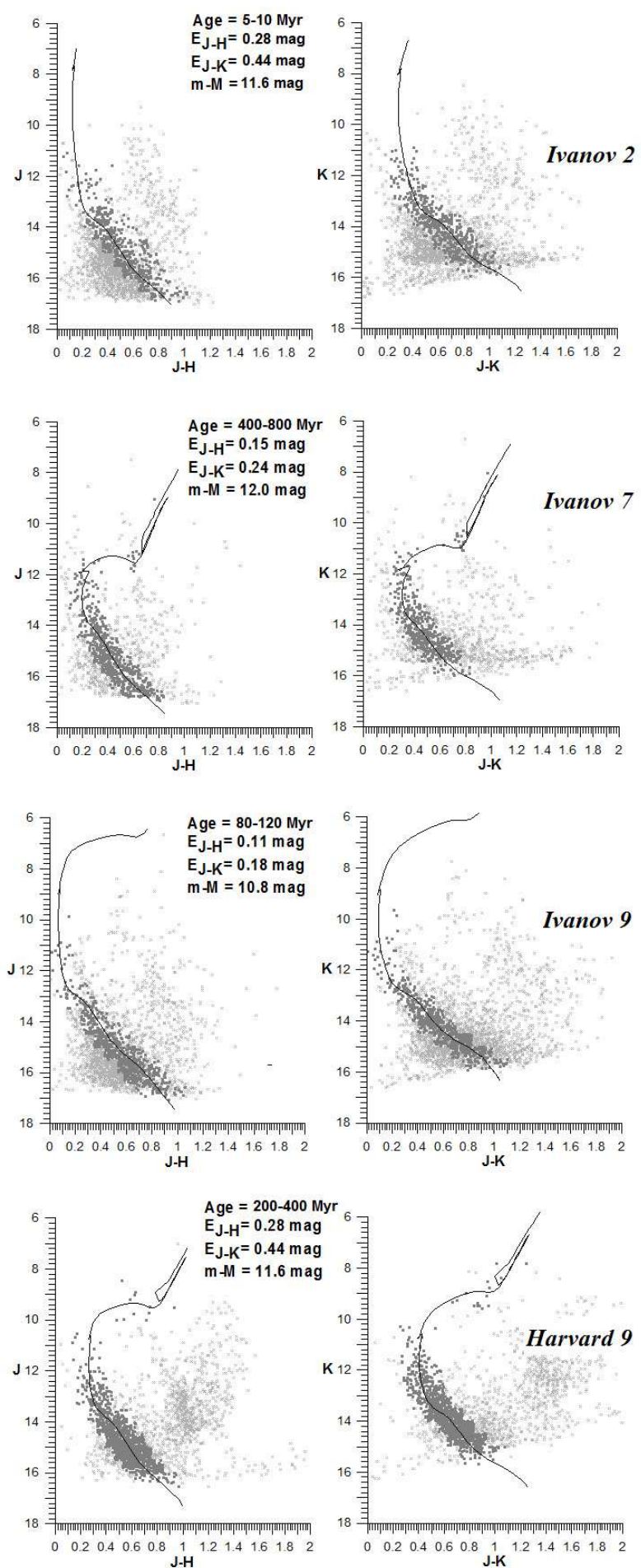

Figure 5. The CMDs of the clusters under investigation. Dark dots represent the probable member stars lying closely to the fitted isochrones curves and light dots refer to the contaminated field stars. Age, color excess and distance modulus are shown in each cluster.

regation, which may affect even poorly populated, relatively young clusters (Scalo 1998). On the other hand, the properties and evolution of a star are closely related to its mass, so the determination of the initial mass function (IMF) is needed. It is an important diagnostic tool for studying large quantities of star clusters. IMF is an empirical relation that describes the mass distribution of a population of stars in terms of their 

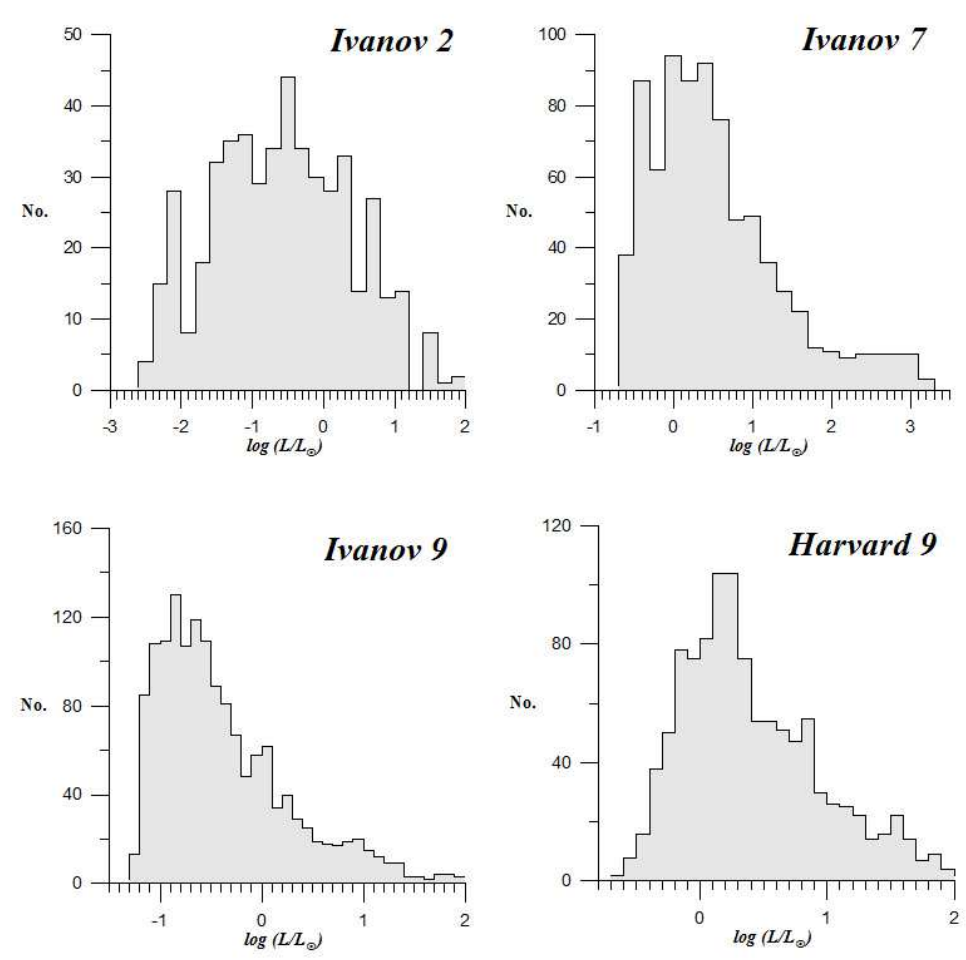

Figure 6. The luminosity functions of the clusters under consideration.
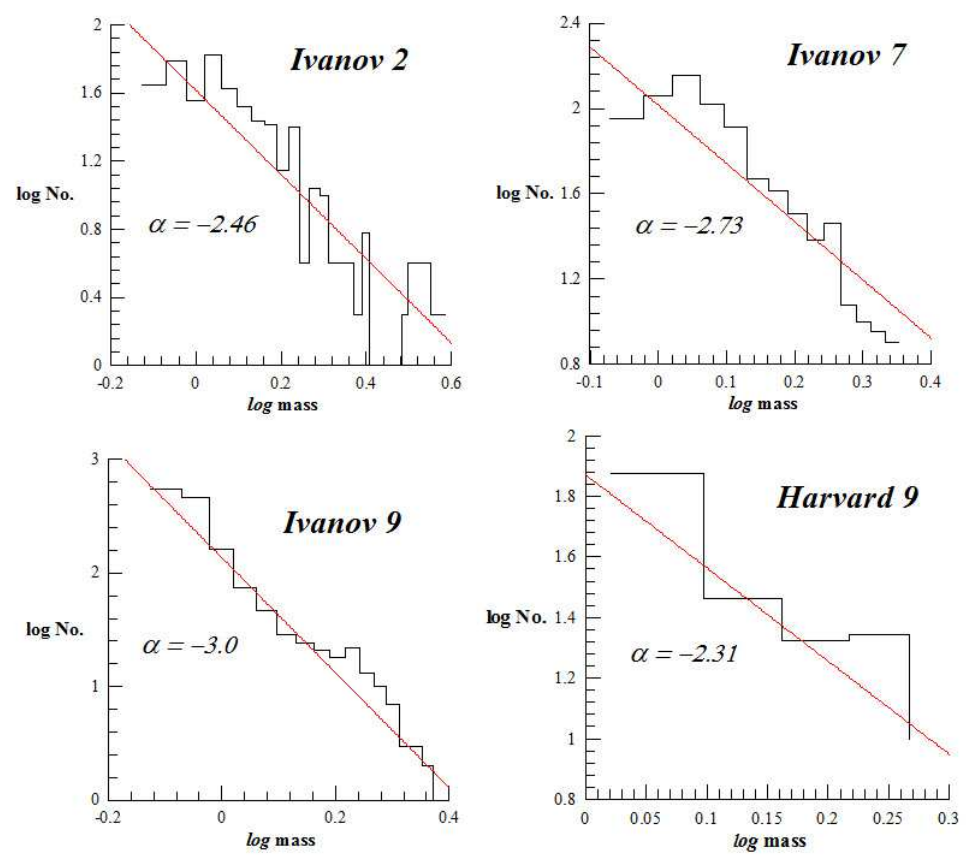

Figure 7. The mass functions of the clusters under consideration; the IMF is shown for each cluster respectively.

theoretical initial mass. The IMF is defined in terms of a power law as follows: where $\frac{d N}{d M}$ is the number of stars of mass interval $(\mathrm{M}: \mathrm{M}+\mathrm{dM})$, and $\alpha$ is a dimensionless exponent. The IMF for massive stars $\left(>1 M_{\odot}\right)$ has been studied and well established by Salpeter (1955), where $\alpha=2.35$. This form of Salpeter shows that the number of stars in 
each mass range decreases rapidly with increasing mass. It is noted that the investigated MF slope ranging of the clusters under consideration are found to be -2.47 , $-2.73,-5.10$ and -2.31 , which are found to be around the Salpeter's value as shown in Figure 7.

To estimate the total mass of the candidate clusters, the mass of each star has been estimated from a polynomial equation developed from the data of the solar metallicity isochrones (absolute magnitudes versus actual masses) at the ages of the clusters. The sum of products of the number of stars in each bin by the mean mass of that bin yields the total mass of the clusters, which are found to be 580, 470, 645 and $1450 M_{\odot}$ for Ivanov 2, 7, 9, and Harvard 9 respectively.

\section{Conclusion}

In the present work, we have analysed the unstudied four open clusters Ivanov 2, 7, 9, and Harvard 9. The astrophysical parameters have been estimated for the first time for these clusters using the PPMXL database of Röser et al. (2010). The main conclusions are summarized and listed in Table 2.

\section{ACKNOWLEDGMENTS}

We thank the anonymous referee for her/his valuable comments and suggestions. It is worthy to mention that, this publication made use of WEBDA, DIAS catalogs, and the data products from the PPMXL database of Röser et al. (2010).

\section{REFERENCES}

Bica, E., Bonatto, C., \& Dutra, C. M. 2003, Does Cyg OB2 Harbour Any Open Cluster?, A\&A, 405, 991

Bonatto, C., \& Bica, E. 2003, Mass Segregation in M 67 with 2MASS, A\&A, 405, 525

Bonatto, Ch., Bica, E., \& Girardi, L. 2004, Theoretical Isochrones Compared to 2MASS Observations: Open Clusters at Nearly Solar Metallicity, A\&A, 415, 571

Bonatto, C., \& Bica, E. 2006, Methods for Improving Open Cluster Fundamental Parameters Applied to M 52 and NGC 3960, A\&A, 455,

Carpenter, J. M. 2001, Color Transformations for the 2MASS Second Incremental Data Release, AJ, 121, 2851

Clariá, J. J., \& Lapasset, E. 1986, Fundamental Parameters of the Open Cluster NGC 2567, AJ, 91, 326

Dutra, C. M., Santiago, B. X., \& Bica, E. 2002, LowExtinction Windows in the Inner Galactic Bulge, A\&A, 381,219

Fiorucci, M., \& Munari, U. 2003, The Asiago Database on Photometric Systems (ADPS). II. Band and Reddening Parameters, A\&A, 401, 781

Gaburov, E., \& Gieles, M. 2008, Mass Segregation in Young Star Clusters - Can It Be Detected from the Integrated Photometric Properties?, MNRAS, 391, 190

Girardi, L., Williams, B. F., Gilbert, K. M., Rosenfield, P., Dalcanton, J., Marigo, P., Boyer, M. L., Dolphin, A., Weisz, D. R., Melbourne, J., et al. 2010, The ACS Nearby Galaxy Survey Treasury. IX. Constraining Asymptotic Giant Branch Evolution with Old Metal-Poor Galaxies, ApJ, 724, 1030
Jeffries, R. D., Thurston, M. R., \& Hambly, N. C. 2001, Photometry and Membership for Low Mass Stars in the Young Open Cluster NGC 2516, A\&A, 375, 863

Khalaj, P., \& Baumgardt, H. 2013, The Stellar Mass Function, Binary Content and Radial Structure of the Open Cluster Praesepe Derived from PPMXL and SDSS Data, MNRAS, 434, 3236

King, I. R. 1966, The Structure of Star Clusters. III. Some Simple Dynamical Models, AJ, 71, 64

Marigo, P., Girardi, L., Bressan, A., Groenewegen, M. A. T., Silva, L., \& Granato, G. L. 2008, Evolution of Asymptotic Giant Branch Stars. II. Optical to Far-Infrared Isochrones with Improved TP-AGB Models, A\&A, 482, 883

Monet, D. G., Levine, S. E., Canzian, B., Ables, H. D., Bird, A. R., Dahn, C. C., et al. 2003, The USNO-B Catalog, AJ, 125, 984

Nilakshi, S. R., Pandey, A. K., \& Mohan, V. 2002, A Study of Spatial Structure of Galactic Open Star Clusters, A\&A, 383, 153

Röser, S., Demleitner, M., \& Schilbach, E. 2010, The PPMXL Catalog of Positions and Proper Motions on the ICRS. Combining USNO-B1.0 and the Two Micron All Sky Survey (2MASS), AJ, 139, 2440

Salpeter, E. E. 1955, The Luminosity Function and Stellar Evolution, ApJ, 121, 161

Scalo, J. 1998, The IMF Revisited: A Case for Variations, ASPC, 142, 201

Schlafly, E. F., \& Finkbeiner, D. P. 2011, Measuring Reddening with Sloan Digital Sky Survey Stellar Spectra and Recalibrating SFD, ApJ, 737, 103

Schlegel, D. J., Finkbeiner, D. P., \& Davis, M. 1998, Maps of Dust Infrared Emission for Use in Estimation of Reddening and Cosmic Microwave Background Radiation Foregrounds, ApJ, 500, 525

Sharma, S., Pandey, A. K., Ogura, K., Mito, H., Tarusawa, K., \& Sagar, R. 2006, Wide-Field CCD Photometry around Nine Open Clusters, AJ, 132, 1669

Skrutskie, M. F., Cutri, R. M., Stiening, R., Weinberg, M. D., Schneider, S., Carpenter, J. M., et al. 2006, The Two Micron All Sky Survey (2MASS), AJ, 131, 1163

Tadross, A. L. 2005, Analytical Studies of NGC 1193, AN, 326,19

Tadross, A. L. 2011, A Catalog of 120 NGC Open Star Clusters, JKAS, 44, 1

Wu, Z.-Y., Ma, J., \& Zhou, X. 2011, Independent Determination of the Systematic and Random Errors of the Proper Motions in the PPMXL Catalogue Using Quasars, PASP, 123, 1313 